\title{
A Criterion For Testing Hypothesis About Impulse Response Function
}

\author{
Yu. V. Kozachenko ${ }^{1}$, I.V. Rozora ${ }^{2, *}$ \\ ${ }^{1}$ Department of Probability Theory, Statistics and Actuarial Mathematics, Faculty of Mathematics and Mechanics, Taras Shevchenko \\ National University of Kyiv,Ukraine, yvk@univ.kiev.ua. \\ ${ }^{2}$ Department of Applied Statistics, Faculty of Cybernetics, Taras Shevchenko National University of Kyiv, Ukraine, irozora@bigmir.net.
}

(Received: 16 April 2016; Accepted: 4 July 2016)

\begin{abstract}
In this paper a time-invariant continuous linear system with a real-valued impulse response function is considered. A new method for the estimator construction of the impulse response function is proposed. Two criteria on the shape of the impulse response function are given.
\end{abstract}

Keywords Impulse response function, cross-correlogram, Hermite polynomials, large deviation probability, rate of convergence, criterion for testing hypothesis.

AMS 2010 subject classifications 62F03, 60G15, 33C45, 60F10.

DOI: $10.19139 /$ soic.v4i3.222

\section{Introduction}

The problem of estimation of a stochastic linear system has been a matter of active research for the last five decades. One of the simplest models considers a black box?with some input and giving a certain output. The input may be single or multiple and there is the same choice for the output. This generates a great amount of models that can be considered. The sphere of applications of these models is vary extensive, ranging from signal processing and automatic control to econometrics (errors-in-variables models). For more details, see [15], [22] and [23].

We are interested in the estimation of the so-called impulse function from observations of responses of a SISO (single-input single-output) system to certain input signals. This problem can be considered both for linear and nonlinear systems. To solve this problem, different statistical approaches were used as well as various deterministic methods that are based on a perturbation of the system by stationary stochastic processes and the further analysis of some characteristics of both input and output processes. Let us mention two monographs on this problem by Bendat and Piersol [3] and Schetzen [22]. Akaike [1] studied a MISO (multiple-input single-output) linear system and obtained estimates of the Fourier transform of the response function in each component. He considered later a scenario involving non-Gaussian processes [2].

Some methods for estimation of unknown impulse response function of linear system and the study of properties of corresponding estimators were considered in the works of Buldygin and his followers. These methods are based on constructing a sample cross-correlogram between the input stochastic process and the response of the system.

Conditions for asymptotic unbiasedness, consistency, asymptotic normality of the integral-type zero-mean crosscorrelogram estimators for the response function in the space of continuous functions were investigated in the papers [9], [10], [4], [5], [6].

\footnotetext{
${ }^{*}$ Correspondence to: 60 Volodymyrska str., Department of Applied Statistics, Faculty of Cybernetics, Taras Shevchenko National University of Kyiv 01601,Ukraine (irozora@bigmir.net).
}

ISSN 2310-5070 (online) ISSN 2311-004X (print)

Copyright (C) 2016 International Academic Press 
The discrete-time sample inputoutput cross-correlogram as estimator of the response function was considered by Buldygin, Utzet, Kurochka and Zaiats [8], [11]. Both asymptotic normality of finite-dimensional distributions of the estimates and their asymptotic normality in spaces of continuous functions were studied. An inequality of the distribution for supremum of estimation error in the space of continuous functions in the case of integral-type cross-correlogram estimator was obtained in [19].

In this paper a time-invariant continuous linear system with a real-valued impulse response function is considered. A new method for the construction of estimator of the impulse response function is proposed.

The paper consists of 7 sections.

In the second Section we describe the main definitions and general properties of the estimator. The input signal process is supposed to be a zero mean Gaussian stochastic process which is represented as a treamed sum with respect to orthonormal basis in $L_{2}(\mathbf{R})$.

In section 3 properties of the Hermite polynomials are described. Since the family of the Hermite functions forms an orthonormal basis in $L_{2}(\mathbf{R})$, then the input process of the system can be represented as a series with respect to the Hermite polynomials. Estimates of mathematical expectation, variance and variance of the increments for the estimator of impulse function are found.

Section 4 deals with square Gaussian random variables and processes. Inequalities for the $C(T)$ norm as well as for $L_{p}(T)$ norm of a square Gaussian stochastic process are shown.

In the fifth section the convergence rate for the estimator of unknown impulse response function in the space of continuous functions and in the space $L_{p}([0, A])$ is investigated.

In the sixth section two criteria are developed on the shape of the impulse response function.

\section{The estimator of an impulse response function and its properties}

Consider a time-invariant continuous linear system with a real-valued square integrable impulse response function $L(\tau), \tau \in \mathbf{R}$. This means that the response of the system to an input signal $X(t), t \in \mathbf{R}$, has the following form:

$$
Y(t)=\int_{0}^{+\infty} L(\tau) X(t-\tau) d \tau
$$

and $L \in L_{2}(\mathbf{R})$. In practice the system is often supposed to be a causal linear system, that is, $L(\tau)=0$ as $\tau<0$. Hereinafter we will consider only such feasible system. Then the system (1) can be written as

$$
Y(t)=\int_{-\infty}^{+\infty} L(\tau) X(t-\tau) d \tau
$$

One of the problems arising in the theory of linear systems is to estimate the function $L$ from observations of responses of the system to certain input signals.

Consider a real-valued Gaussian zero mean stochastic process $X_{N}=\left(X_{N, t}(u), u \in \mathbf{R}\right)$, that can be presented as

$$
X_{N}(u)=X_{t, N}(u)=\sum_{k=1}^{N} \xi_{k} \varphi_{k}(t-u),
$$

where a fixed value $t>0$, the system of functions $\left\{\varphi_{k}(t), k=\overline{1, \infty}\right\}$ is an orthonormal basis (ONB) in $L_{2}(\mathbf{R})$ and random variables $\xi_{k}, k \geq 1$, are independent with $\mathbf{E} \xi_{k}=0, \mathbf{E} \xi_{k} \xi_{l}=\delta_{k l}$, where $\delta_{k l}$ is a Kronecker symbol.

Let us denote

$$
a_{k}=\int_{0}^{+\infty} L(\tau) \varphi_{k}(\tau) d \tau
$$

If the system (1) is perturbed by the stochastic process $X_{N}$, then for the output process we obtain 


$$
\begin{aligned}
Y_{N}(t) & =\int_{0}^{+\infty} L(\tau) X_{N}(t-\tau) d \tau \\
& =\sum_{k=1}^{N} \xi_{k} \int_{0}^{+\infty} L(\tau) \varphi_{k}(\tau) d \tau=\sum_{k=1}^{N} \xi_{k} a_{k} .
\end{aligned}
$$

Consider the sequence of independent copies $\left\{X_{N, i}(u), i=1, \ldots, n\right\}$ of the Gaussian process (2), that perturb the system (1). That is,

$$
X_{N, i}(u)=\sum_{k=1}^{N} \xi_{k, i} \varphi_{k}(t-u),
$$

where $\xi_{k, i}$ are independent normal distributed random variables, $\mathbf{E} \xi_{k, i}=0$ and $E \xi_{k, i} \xi_{l, j}=\delta_{k l} \delta_{i j}, k, l=$ $\overline{1, N}, i, j=\overline{1, n}$.

By $\left\{Y_{N, i}(t), i=1, \ldots, n\right\}$ denote the reactions of the system on input signals $\left\{X_{N, i}(u)\right\}$.

An estimator for impulse function $L$ at the point $\tau, \tau>0$, is defined by

$$
\hat{L}_{N, n}(\tau)=\frac{1}{n} \sum_{i=1}^{n} Y_{N, i}(\tau) X_{N, i}(t-\tau), \tau>0 .
$$

Since $L \in L_{2}(\mathbf{R})$, then the following remarks hold true.

Remark 2.1. The integral in (1) is considered as the mean-square Riemann integral.

The integral in (1) exists if and only if there exists the Riemann integral (see [14], p. 278)

$$
\int_{0}^{\infty} \int_{0}^{\infty} L(\tau) K_{X_{N}}(s, \tau) L(s) d s d \tau
$$

Since the covariance function of the process $X_{N}$ is

$$
K_{X_{N}}(s, \tau)=\sum_{k=1}^{N} \varphi_{k}(t-s) \varphi_{k}(t-\tau)
$$

and $\left\{\varphi_{k}(t), k \geq 1\right\}$ is an orthonormal basis in $L_{2}(\mathbf{R})$ and $L \in L_{2}(\mathbf{R})$, then the integral (7) exists. Therefore, there exists also the integral in (1).

Remark 2.2. The process $X_{N, i}(t-\tau)$ in (6) depends only on $\tau$ and doesn't depend on $t$. It follows from the definition of the process in (5).

\section{Lemma 2.1}

The following relations hold true:

$$
\mathbf{E} \hat{L}_{N, n}(\tau)=\sum_{k=1}^{N} \varphi_{k}(\tau) a_{k}, \quad \tau>0,
$$

and

$$
L(\tau)-\mathbf{E} \hat{L}_{N, n}(\tau)=\sum_{k=N+1}^{\infty} \varphi_{k}(\tau) a_{k}, \quad \tau>0 .
$$


Proof

The joint covariance function the processes $X_{N, i}$ and $Y_{N, i}$ equals

$$
\begin{aligned}
\mathbf{E}\left(Y_{N, i}(\tau) X_{N, i}(t-\tau)\right) & =\mathbf{E} \sum_{k=1}^{N} \sum_{l=1}^{N} \varphi_{k}(\tau) a_{l} \xi_{k, i} \xi_{l, i} \\
& =\sum_{k=1}^{N} \varphi_{k}(\tau) a_{k} .
\end{aligned}
$$

From the equality above it follows that

$$
\mathbf{E} \hat{L}_{N, n}(\tau)=\frac{1}{n} \sum_{i=1}^{n} \mathbf{E}\left(Y_{N, i}(\tau) X_{N, i}(t-\tau)\right)=\sum_{k=1}^{N} \varphi_{k}(\tau) a_{k} .
$$

Therefore, relation (8) is proved.

Since $L \in L_{2}(\mathbf{R})$, then the function $L$ can be expanded into the series by orthonormal basis $\left\{\varphi_{k}(t), k \geq 1\right\}$. We obtain

$$
L(\tau)=\sum_{k=1}^{\infty} \varphi_{k}(\tau) \cdot \int_{0}^{+\infty} L(\tau) \varphi_{k}(\tau) d \tau=\sum_{k=1}^{\infty} \varphi_{k}(\tau) \cdot a_{k}
$$

where $a_{k}$ is from (3).

Remark 2.3. The series (11) converges in the mean square sense.

Equalities (11) and (8) imply that

$$
L(\tau)-\mathbf{E} \hat{L}_{N, n}(\tau)=\sum_{k=N+1}^{\infty} \varphi_{k}(\tau) \cdot a_{k}, \quad \tau>0 .
$$

We prove now the following auxiliary Lemma.

Lemma 2.2

The joint moments of $\hat{L}_{T, N}$ is equal to

$$
\begin{aligned}
\mathbf{E} \hat{L}_{N, n}(\tau) \hat{L}_{N, n}(\theta) & =\frac{1}{n} \sum_{k=1}^{N} \sum_{l=1}^{N}\left(n a_{k} a_{l} \varphi_{k}(\tau) \varphi_{l}(\theta)+a_{k}^{2} \varphi_{l}(\tau) \varphi_{l}(\theta)\right. \\
& \left.+a_{k} a_{l} \varphi_{l}(\tau) \varphi_{k}(\theta)\right),
\end{aligned}
$$

where the coefficients $a_{k}$ are defined in (3).

Proof

By definition of estimator $\hat{L}_{T, N}(6)$ and the values of the processes $X_{N, i}$ from (5) and $Y_{N, i}$ from (4) we have

$$
\begin{aligned}
\mathbf{E} \hat{L}_{N, n}(\tau) \hat{L}_{N, n}(\theta) & =\frac{1}{n^{2}} \sum_{i=1}^{n} \sum_{j=1}^{n} \mathbf{E} Y_{N, i}(\tau) X_{N, i}(t-\tau) Y_{N, j}(\theta) X_{N, j}(t-\theta) \\
& =\frac{1}{n^{2}} \sum_{i=1}^{n} \sum_{j=1}^{n} \sum_{k, l, u, v=1}^{N} a_{k} a_{u} \varphi_{l}(\tau) \varphi_{v}(\theta) \mathbf{E} \xi_{k, i} \xi_{l, i} \xi_{u, j} \xi_{v, j}
\end{aligned}
$$


To prove the assertion it's enough to find $\mathbf{E} \xi_{k, i} \xi_{l, i} \xi_{u, j} \xi_{v, j}$. Using the Isserlis?formula for the centered Gaussian random variables [7] we obtain:

$$
\begin{aligned}
\mathbf{E} \xi_{k, i} \xi_{l, i} \xi_{u, j} \xi_{v, j} & =\mathbf{E} \xi_{k, i} \xi_{l, i} \mathbf{E} \xi_{u, j} \xi_{v, j} \\
& +\mathbf{E} \xi_{k, i} \xi_{u, j} \mathbf{E} \xi_{l, i} \xi_{v, j} \\
& +\mathbf{E} \xi_{k, i} \xi_{v, j} \mathbf{E} \xi_{l, i} \xi_{u, j}
\end{aligned}
$$

By the definition of the processes $X_{N, i}$ and $Y_{N, i}$, we have

$$
\begin{array}{rll}
\mathbf{E} \xi_{k, i} \xi_{l, i} & =\delta_{k l}, & \mathbf{E} \xi_{u, j} \xi_{v, j}=\delta_{u v}, \\
\mathbf{E} \xi_{k, i} \xi_{u, j}=\delta_{k u} \delta_{i j}, & \mathbf{E} \xi_{l, i} \xi_{v, j}=\delta_{l v} \delta_{i j}, \\
\mathbf{E} \xi_{k, i} \xi_{v, j}=\delta_{k v} \delta_{i j}, & \mathbf{E} \xi_{l, i} \xi_{u, j}=\delta_{l u} \delta_{i j},
\end{array}
$$

(12) will be completely proved if the equalities above and (14) will be substituted in (13).

The next Corollary follows from Lemma 2.2.

\section{Corollary 2.1}

The variance of the estimator $\hat{L}_{N, n}$ is equal to

$$
\begin{aligned}
\operatorname{Var} \hat{L}_{N, n}(\tau) & =\mathbf{E} \hat{L}_{N, n}^{2}(\tau)-\left(\mathbf{E} \hat{L}_{N, n}(\tau)\right)^{2} \\
& =\frac{1}{n} \sum_{l=1}^{N} \sum_{k=1}^{N}\left(a_{k}^{2} \varphi_{l}^{2}(\tau)+a_{k} a_{l} \varphi_{k}(\tau) \varphi_{l}(\tau)\right) .
\end{aligned}
$$

$$
\operatorname{Var}\left(\hat{L}_{N, n}(\tau)-\hat{L}_{N, n}(\theta)\right)=\operatorname{Var} \hat{L}_{N, n}(\tau)+\operatorname{Var} \hat{L}_{N, n}(\theta)-2 \operatorname{cov}\left(\hat{L}_{N, n}(\tau), \hat{L}_{N, n}(\theta)\right)
$$

Lemma 2.3

Suppose that the Lipschitz condition with a rate $\alpha \in(0,1]$ for the functions $\varphi_{k}(t)$ holds on a segment $[0, A]$. It means that there exist constants $c_{k, \varphi}$ such that

$$
\left|\varphi_{k}(t)-\varphi_{k}(s)\right| \leq c_{k, \varphi}|t-s|^{\alpha} \quad \text { for all } \quad t, s \in[0, A] \quad \text { and } \quad k \geq 1 .
$$

In this case

$$
\operatorname{Var}\left(\hat{L}_{N, n}(\tau)-\hat{L}_{N, n}(\theta)\right) \leq C(N, n)|\tau-\theta|^{\alpha}, \quad \alpha \in(0,1], \tau, \theta \in[0, A]
$$

where

$$
C(N, n)=\frac{1}{n} \sum_{k=1}^{N} \sum_{l=1}^{N}\left(a_{k}^{2}\left(\left|\varphi_{l}(\tau)\right|+\left|\varphi_{l}(\theta)\right|\right) c_{l, \varphi}+a_{k} a_{l}\left(\left|\varphi_{l}(\tau)\right| c_{k, \varphi}+\left|\varphi_{k}(\theta)\right| c_{l, \varphi}\right)\right)
$$

Proof

From relations (8) and (12) it follows that

$$
\begin{aligned}
\operatorname{cov}\left(\hat{L}_{N, n}(\tau), \hat{L}_{N, n}(\theta)\right) & =\mathbf{E} \hat{L}_{N, n}(\tau) \hat{L}_{N, n}(\theta)-\mathbf{E} \hat{L}_{N, n}(\tau) \mathbf{E} \hat{L}_{N, n}(\theta) \\
& =\frac{1}{n} \sum_{k=1}^{N} \sum_{l=1}^{N}\left(a_{k}^{2} \varphi_{l}(\tau) \varphi_{l}(\theta)+a_{k} a_{l} \varphi_{l}(\tau) \varphi_{k}(\theta)\right),
\end{aligned}
$$

Therefore, by (15), (16) and (19), making elementary reduction we obtain

$$
\begin{aligned}
\operatorname{Var}\left(\hat{L}_{N, T}(\tau)-\hat{L}_{N, T}(\theta)\right) & =\frac{1}{n} \sum_{k, l=1}^{N}\left(a_{k}^{2}\left(\varphi_{l}(\tau)\left(\varphi_{l}(\tau)-\varphi_{l}(\theta)\right)+\varphi_{l}(\theta)\left(\varphi_{l}(\theta)-\varphi_{l}(\tau)\right)\right)\right. \\
& \left.+a_{k} a_{l}\left(\varphi_{l}(\tau)\left(\varphi_{k}(\tau)-\varphi_{k}(\theta)\right)+\varphi_{k}(\theta)\left(\varphi_{l}(\theta)-\varphi_{l}(\tau)\right)\right)\right)
\end{aligned}
$$

The Lemma will be fully proved if in (20) the Lipschitz condition (17) for the function $\varphi_{k}(\tau), \tau \in[0, A]$, will be used. 


\section{The use of Hermite polynomials for the presentation of the estimator}

Consider the Hermite polynomials of the degree $n \geq 1$ :

$$
H_{n}(x)=(-1)^{n} e^{\frac{x 2}{2}} \frac{d^{n}}{d x^{n}}\left(e^{-\frac{x^{2}}{2}}\right) .
$$

It is shown in the book [13] that

$$
\frac{d^{k} H_{n}(x)}{d x^{k}}=n(n-1)(n-2) \cdots(n-k+1) H_{n-k}(x) .
$$

In particular case $k=1$ we have

$$
\frac{d H_{n}(x)}{d x}=n H_{n-1}(x)
$$

It is known that the system of the Hermite functions

$$
g_{n}(x)=\frac{e^{-\frac{x^{2}}{4}} H_{n}(x)}{\sqrt{n !}(2 \pi)^{1 / 4}}=\frac{(-1)^{n} e^{\frac{x^{2}}{4}}}{\sqrt{n !}(2 \pi)^{1 / 4}} \frac{d^{n}}{d x^{n}}\left(e^{-\frac{x^{2}}{2}}\right), \quad n \geq 0,
$$

is an orthonormal basis (ONB) in $L_{2}(\mathbf{R})$ (see, for example, [13]).

Suppose now that the input signal processes of the system (1) are zero mean Gaussian stochastic processes that are formed by the Hermite ONB (22). This means that the processes $X_{N, i}(u), i=\overline{1, n}$, are represented in such way:

$$
X_{N, i}(u)=\sum_{k=1}^{N} \xi_{k, i} g_{k}(t-u) \quad \text { for } t, u \in \mathbf{R} .
$$

It follows from (1) that the output processes $Y_{N, i}(t), t \in \mathbf{R}$, equal

$$
Y_{N, i}(t)=\int_{0}^{+\infty} L(\tau) X_{N, i}(t-\tau) d \tau, \quad \text { for } \quad i=\overline{1, n} .
$$

The estimator for impulse function $L$ in the point $\tau, \tau>0$, is defined by (6).

Consider the following conditions:

Condition A. There exists an integral

$$
\int_{-\infty}^{+\infty} Z_{L}(z) d z<\infty
$$

where the function $Z_{L}(z)$ is equal to

$$
Z_{L}(z)=\frac{d^{3} L(z)}{d z^{3}}-\frac{3 z}{2} \frac{d^{2} L(z)}{d z^{2}}+\frac{3 z^{2}-6}{4} \frac{d L(z)}{d z}+\frac{6 z-z^{3}}{8} L(z) .
$$

Denote

$$
I_{L}=\left|\int_{-\infty}^{+\infty} Z_{L}(z) d z\right|
$$

Condition B. The function $L(z)$ increases on $z$ no faster than $e^{z^{2} / 4}$. It means that there exists a constant $c \in\left(0, \frac{1}{4}\right)$ that

$$
L(z) e^{-c z^{2}} \rightarrow 0, z \rightarrow+\infty .
$$

The following Lemma gives an estimate for $\left|L(\tau)-\mathbf{E} \hat{L}_{N, n}(\tau)\right|$ and for the variance $\operatorname{Var}\left(\hat{L}_{T, N}(\tau)\right)$. 
Lemma 3.1

Assume that the conditions $\mathbf{A}$ and $\mathbf{B}$ are satisfied. Then

$$
\begin{gathered}
\left|L(\tau)-\mathbf{E} \hat{L}_{N, n}(\tau)\right| \leq \frac{2 K^{2}}{3 \sqrt{N+1}} I_{L}, \\
\operatorname{Var} \hat{Z}_{N, n}(\tau)<\frac{K^{4} I_{L}^{2}}{n}\left(N \frac{N^{2}-1}{2 N^{2}}+\frac{(2 \sqrt{N}-2)^{2}}{N}\right),
\end{gathered}
$$

where the number $K=1,086435$.

Proof

From (9) it follows that

$$
L(\tau)-\mathbf{E} \hat{L}_{N, n}(\tau)=\sum_{k=N+1}^{\infty} g_{k}(\tau) a_{k}
$$

Calculate the required values $a_{k}$ by using equality (21) and partial integration:

$$
\begin{aligned}
a_{k} & =\int_{-\infty}^{+\infty} L(z) g_{k}(z) d z \\
& =\int_{-\infty}^{+\infty} \frac{L(z) e^{-\frac{z^{2}}{4}} H_{k}(z)}{\sqrt{k !}(2 \pi)^{1 / 4}} d z \\
& =\frac{1}{\sqrt{k+1}} \int_{-\infty}^{+\infty} \frac{L(z) e^{-\frac{z^{2}}{4}}}{\sqrt{(k+1) !(2 \pi)^{1 / 4}}} \frac{d H_{k+1}(z)}{d z} d z \\
& =\frac{1}{\sqrt{k+1}}\left(\left.\frac{L(z) e^{-\frac{z^{2}}{4}} H_{k+1}(z)}{\sqrt{(k+1) !}(2 \pi)^{1 / 4}}\right|_{-\infty} ^{+\infty}-\int_{-\infty}^{+\infty} \frac{d\left(L(z) e^{-\frac{z^{2}}{4}}\right)}{d z} \frac{H_{k+1}(z)}{\sqrt{(k+1) !(2 \pi)^{1 / 4}}} d z\right)
\end{aligned}
$$

Since $H_{k}(z) e^{-z^{2} / 4}$ tends to zero as $z \rightarrow \pm \infty$, and by condition A the function $L(z)$ increases on $z$ no faster than $e^{z^{2} / 4}$, then

$$
a_{k}=-\frac{1}{\sqrt{k+1}} \int_{-\infty}^{+\infty} \frac{d\left(L(z) e^{-\frac{z^{2}}{4}}\right)}{d z} \frac{H_{k+1}(z)}{\sqrt{(k+1) !}(2 \pi)^{1 / 4}} d z
$$

Let's apply the integration by parts two times more. We obtain

$$
\begin{aligned}
a_{k} & =-\frac{1}{\sqrt{(k+1)(k+2)}} \int_{-\infty}^{+\infty} \frac{d\left(L(z) e^{-\frac{z^{2}}{4}}\right)}{d z} \frac{d H_{k+2}(z)}{d z} \frac{1}{\sqrt{(k+2) !}(2 \pi)^{1 / 4}} d z \\
& =-\left.\frac{1}{\sqrt{(k+1)(k+2)}} \frac{d\left(L(z) e^{-\frac{z^{2}}{4}}\right)}{d z} \frac{H_{k+2}(z)}{\sqrt{(k+2) !(2 \pi)^{1 / 4}}}\right|_{-\infty} ^{+\infty} \\
& +\frac{1}{\sqrt{(k+1)(k+2)}} \int_{-\infty}^{+\infty} \frac{d^{2}\left(L(z) e^{-\frac{z^{2}}{4}}\right)}{d z^{2}} \frac{H_{k+2}(z)}{\sqrt{(k+2) !}(2 \pi)^{1 / 4}} d z \\
& =\frac{1}{\sqrt{(k+1)(k+2)}} \int_{-\infty}^{+\infty} \frac{d^{2}\left(L(z) e^{-\frac{z^{2}}{4}}\right)}{d z^{2}} \frac{H_{k+2}(z)}{\sqrt{(k+2) !}(2 \pi)^{1 / 4}} d z \\
& =\frac{1}{\sqrt{(k+1)(k+2)(k+3)}} \int_{-\infty}^{+\infty} \frac{d^{3}\left(L(z) e^{-\frac{z^{2}}{4}}\right)}{d z^{3}} \frac{H_{k+3}(z)}{\sqrt{(k+3) !}(2 \pi)^{1 / 4}} d z
\end{aligned}
$$




$$
=\frac{1}{\sqrt{(k+1)(k+2)(k+3)}} \int_{-\infty}^{+\infty} Z_{L}(z) g_{k+3}(z) d z,
$$

where $g_{k+3}(z)$ is the Hermite function of and the function $Z_{L}(z)$ is from (25).

By using (28) and (29) we obtain that

$$
\begin{aligned}
L(\tau)-\mathbf{E} \hat{L}_{N, n}(\tau) & =\sum_{k=N+1}^{\infty} g_{k}(\tau) a_{k} \\
& =\sum_{k=N+1}^{\infty} \frac{g_{k}(\tau)}{\sqrt{(k+1)(k+2)(k+3)}} \int_{-\infty}^{+\infty} Z_{L}(z) g_{k+3}(z) d z
\end{aligned}
$$

From the Cramér inequality [13] it follows that for all $z \in \mathbf{R}\left|g_{k}(z)\right|<K$, where $K=1,086435$. Therefore, the coefficients $a_{k}$ can be estimated as follows

$$
\left|a_{k}\right| \leq \frac{K}{\sqrt{(k+1)(k+2)(k+3)}}\left|\int_{-\infty}^{+\infty} Z_{L}(z) d z\right| .
$$

From relations (30) and (31) it follows that

$$
\left|L(\tau)-\mathbf{E} \hat{L}_{N, n}(\tau)\right| \leq K^{2} I_{L} \sum_{k=N+1}^{\infty} \frac{1}{\sqrt{(k+1)(k+2)(k+3)}}
$$

Evaluate now the sum in (32).

$$
\begin{aligned}
\sum_{k=N+1}^{\infty} \frac{1}{\sqrt{(k+1)(k+2)(k+3)}} & <\sum_{k=N+1}^{\infty} \frac{1}{\sqrt{(k+1)^{3}}} \\
& =\sum_{k=N+1}^{\infty} \int_{k}^{k+1} \frac{1}{\sqrt{(k+1)^{3}}} d x<\sum_{k=N+1}^{\infty} \int_{k}^{k+1} \frac{1}{x^{3 / 2}} d x \\
& =\int_{N+1}^{\infty} \frac{1}{x^{3 / 2}} d x=\frac{2}{3 \sqrt{N+1}} .
\end{aligned}
$$

Hence, inequalities (32) and (33) imply (26).

Find the estimate for the variance of the process $\hat{Z}_{N, n}(\tau)$. From (15) follows that

$$
\operatorname{Var} \hat{Z}_{N, n}(\tau)=\frac{1}{n} \sum_{l=1}^{N} \sum_{k=1}^{N}\left(a_{k}^{2} g_{l}^{2}(\tau)+a_{k} a_{l} g_{k}(\tau) g_{l}(\tau)\right)
$$

Since $\left|g_{k}(\tau)\right|<K$ and using inequality (31) for $a_{k}$, we obtain that

$$
\begin{aligned}
\operatorname{Var} \hat{Z}_{N, n}(\tau) & <\frac{K^{4} I_{L}^{2}}{n} \sum_{l=1}^{N} \sum_{k=1}^{N}\left(\frac{1}{(k+1)(k+2)(k+3)}\right. \\
& \left.+\frac{1}{\sqrt{(k+1)(k+2)(k+3)} \sqrt{(l+1)(l+2)(l+3)}}\right) \\
& =\frac{K^{4} I_{L}^{2}}{n}\left(N \sum_{k=1}^{N} \frac{1}{(k+1)(k+2)(k+3)}+\left(\sum_{k=1}^{N} \frac{1}{\sqrt{(k+1)(k+2)(k+3)}}\right)^{2}\right) .
\end{aligned}
$$


Similarly to (33) the sums above can be evaluated in the following way

$$
\begin{aligned}
& \sum_{k=1}^{N} \frac{1}{(k+1)(k+2)(k+3)}<\sum_{k=1}^{N} \frac{1}{(k+1)^{3}} \\
&=\sum_{k=1}^{N-1} \int_{k}^{k+1} \frac{1}{(k+1)^{3}} d x<\sum_{k=1}^{N-1} \int_{k}^{k+1} \frac{1}{x^{3}} d x \\
&=\int_{1}^{N} \frac{1}{x^{3}} d x=\frac{N^{2}-1}{2 N^{2}} ; \\
& \sum_{k=1}^{N} \frac{1}{\sqrt{(k+1)(k+2)(k+3)}}<\int_{1}^{N} \frac{1}{x^{3 / 2}} d x=\frac{2 \sqrt{N}-2}{\sqrt{N}} .
\end{aligned}
$$

If substitute (35), (36) into (34), then we obtain (27).

The following Lemma gives an estimate for the difference of the Hermite functions $\left|g_{k}(x)-g_{k}(y)\right|$ as $x, y \in$ $[0, A]$.

\section{Lemma 3.2}

Let $g_{k}(x), k \geq 1$, be the Hermite functions from (22). Assume that the conditions of Lemma 3.1 are satisfied. Then for any $x, y \in[0, A]$, where $A>0$ is some constant, the inequality

$$
\left|g_{k}(x)-g_{k}(y)\right| \leq c_{k, g}|x-y|^{\alpha}, \quad \alpha \in(0,1],
$$

holds true, where the value $c_{k, g}$ is equal to

$$
\begin{aligned}
c_{k, g} & =\frac{1}{\sqrt{k !}(2 \pi)^{1 / 4}}\left(\frac{2^{\frac{k-\alpha}{2}+1} e^{\frac{A^{2}}{4}}}{\sqrt{\pi}} \Gamma\left(\frac{k+\alpha+1}{2}\right)+\frac{2^{\frac{k}{2}-\alpha} A^{\alpha}}{\sqrt{\pi}} \Gamma\left(\frac{k+1}{2}\right) e^{\frac{A^{2}}{2}}\right) \\
& =\frac{2^{\frac{k-\alpha}{2}-\frac{1}{4}} e^{\frac{A^{2}}{2}}}{\sqrt{k !} \pi^{3 / 4}}\left(2 \Gamma\left(\frac{k+\alpha+1}{2}\right)+2^{-\frac{\alpha}{2}} A^{\alpha} \Gamma\left(\frac{k+1}{2}\right)\right),
\end{aligned}
$$

where $\Gamma(r)=\int_{0}^{\infty} t^{r-1} e^{-t} d t$ is an Euler gamma function.

Proof

Without limiting the generality assume that $x>y \geq 0$. By the definition of the Hermite function:

$$
\begin{aligned}
\left|g_{k}(x)-g_{k}(y)\right| & =\left|\frac{e^{\frac{-x^{2}}{4}}}{\sqrt{k !}(2 \pi)^{1 / 4}} H_{k}(x)-\frac{e^{\frac{-y^{2}}{4}}}{\sqrt{k !}(2 \pi)^{1 / 4}} H_{k}(y)\right| \\
& =\frac{1}{\sqrt{k !}(2 \pi)^{1 / 4}}\left|e^{\frac{x^{2}}{4}} \frac{d^{k}}{d x^{k}}\left(e^{-\frac{x^{2}}{2}}\right) \pm e^{\frac{x^{2}}{4}} \frac{d^{k}}{d y^{k}}\left(e^{-\frac{y^{2}}{2}}\right)-e^{\frac{y^{2}}{4}} \frac{d^{k}}{d y^{k}}\left(e^{-\frac{y^{2}}{2}}\right)\right| \\
& \leq \frac{1}{\sqrt{k !}(2 \pi)^{1 / 4}}\left(e^{\frac{x^{2}}{4}}\left|\frac{d^{k}}{d x^{k}}\left(e^{-\frac{x^{2}}{2}}\right)-\frac{d^{k}}{d y^{k}}\left(e^{-\frac{y^{2}}{2}}\right)\right|+\left|\frac{d^{k}}{d y^{k}}\left(e^{-\frac{y^{2}}{2}}\right)\right|\left|e^{\frac{x^{2}}{4}}-e^{\frac{y^{2}}{4}}\right|\right) \\
& =\frac{1}{\sqrt{k !}(2 \pi)^{1 / 4}}\left(I_{1}+I_{2}\right) .
\end{aligned}
$$

To estimate the values $I_{1}$ and $I_{2}$ let's use the next formula

$$
e^{-\frac{x^{2}}{2}}=\frac{1}{\sqrt{2 \pi}} \int_{-\infty}^{+\infty} e^{i u x-\frac{u^{2}}{2}} d u
$$


that is, actually, a characteristic function for a standard Gaussian random variable. By (40) we have

$$
\frac{d^{k} e^{-\frac{x^{2}}{2}}}{d x^{k}}=\frac{1}{\sqrt{2 \pi}} \int_{-\infty}^{+\infty}(i u)^{k} e^{i u x-\frac{u^{2}}{2}} d u .
$$

From (41) follows that the the quantity $I_{1}$ in the case of $x, y \in[0, A]$ is bounded in a such way:

$$
\begin{aligned}
I_{1} & \leq e^{\frac{A^{2}}{4}}\left|\frac{1}{\sqrt{2 \pi}} \int_{-\infty}^{+\infty}(i u)^{k} e^{i u x-\frac{u^{2}}{2}} d u-\frac{1}{\sqrt{2 \pi}} \int_{-\infty}^{+\infty}(i u)^{k} e^{i u y-\frac{u^{2}}{2}} d u\right| \\
& \leq \frac{e^{\frac{A^{2}}{4}}}{\sqrt{2 \pi}} \int_{-\infty}^{+\infty}|u|^{k}\left|e^{i u x}-e^{i u y}\right| e^{-\frac{u^{2}}{2}} d u
\end{aligned}
$$

Since

$$
\left|e^{i u x}-e^{i u y}\right|=2\left|\sin \left(\frac{u}{2}(x-y)\right)\right|,
$$

then

$$
I_{1} \leq \frac{2 e^{\frac{A^{2}}{4}}}{\sqrt{2 \pi}} \int_{-\infty}^{+\infty}|u|^{k}\left|\sin \left(\frac{u}{2}(x-y)\right)\right| e^{-\frac{u^{2}}{2}} d u
$$

For $u \geq 0$ and $v>0$ the inequality holds true (see, for example, [18])

$$
\left|\sin \frac{u}{v}\right| \leq \frac{u^{\alpha}}{v^{\alpha}}, \quad \alpha \in(0,1]
$$

If (43) will be used for the value (42), then we obtain that

$$
\begin{aligned}
I_{1} & \leq \frac{2 e^{\frac{A^{2}}{4}}}{2^{\alpha} \sqrt{2 \pi}}|x-y|^{\alpha} \int_{-\infty}^{+\infty}|u|^{k+\alpha} e^{-\frac{u^{2}}{2}} d u \\
& =\frac{2^{\frac{k-\alpha}{2}+1} e^{\frac{A^{2}}{4}}}{\sqrt{\pi}} \Gamma\left(\frac{k+\alpha+1}{2}\right)|x-y|^{\alpha}, \quad \alpha \in(0,1] .
\end{aligned}
$$

Remind that $\Gamma(r)=\int_{0}^{\infty} t^{r-1} e^{-t} d t$ is an Euler gamma function.

From (41) follows that

$$
\begin{aligned}
\left|\frac{d^{k} e^{-\frac{y^{2}}{2}}}{d y^{k}}\right| & \leq \frac{1}{\sqrt{2 \pi}} \int_{-\infty}^{+\infty}|u|^{k} e^{-\frac{u^{2}}{2}} d u \\
& =\frac{2^{\frac{k}{2}}}{\sqrt{\pi}} \Gamma\left(\frac{k+1}{2}\right) .
\end{aligned}
$$

Estimate now the value $I_{2}$ from (39). By (45) we have

$$
\begin{aligned}
I_{2} & =\left|\frac{d^{k} e^{-\frac{y^{2}}{2}}}{d y^{k}}\right|\left|e^{\frac{x^{2}}{4}}-e^{\frac{y^{2}}{4}}\right| \\
& \leq \frac{2^{\frac{k}{2}}}{\sqrt{\pi}} \Gamma\left(\frac{k+1}{2}\right) e^{\frac{x^{2}}{2}}\left|1-e^{-\frac{x^{2}-y^{2}}{4}}\right| .
\end{aligned}
$$

Since for $t>0$

$$
1-e^{-t} \leq t^{\alpha}, \quad \alpha \in(0,1]
$$


then for $x>y \geq 0, x, y \in[0, A]$, we obtain

$$
\begin{aligned}
I_{2} & \leq \frac{2^{\frac{k}{2}}}{\sqrt{\pi}} \Gamma\left(\frac{k+1}{2}\right) e^{\frac{x^{2}}{2}}\left|\frac{x^{2}-y^{2}}{4}\right|^{\alpha} \\
& \leq \frac{2^{\frac{k}{2}-\alpha} A^{\alpha}}{\sqrt{\pi}} \Gamma\left(\frac{k+1}{2}\right) e^{\frac{A^{2}}{2}}|x-y|^{\alpha} .
\end{aligned}
$$

If we substitute (44) and (47) into (39), we will have that (37) completely proved.

\section{Corollary 3.1}

From conditions of Lemma 3.1 it follows that

$$
\operatorname{Var}\left(\hat{Z}_{N, n}(\tau)-\hat{Z}_{N, n}(\theta)\right) \leq C_{g}(N, n)|\tau-\theta|^{\alpha}, \quad \alpha \in(0,1], \tau, \theta \in[0, A],
$$

where

$$
C_{g}(N, n)=\frac{K^{3} I_{L}^{2}}{n}\left(2 \frac{N^{2}-1}{2 N^{2}} \sum_{l=1}^{N} c_{l, g}+\sum_{k=1}^{N} \frac{c_{k, g}}{\sqrt{(k+1)(k+2)(k+3)}}\right)
$$

Proof

The assertion (48) follows from Lemmas 2.3 and 3.2.

\section{Square Gaussian random variables and processes}

In this section the definition and some properties of square Gaussian random variables and processes are presented. Let $(\Omega, L, P)$ be a probability space and let $(\mathbf{T}, \rho)$ be a compact metric space with metric $\rho$.

Definition 4.1. [7] Let $\Xi=\left\{\xi_{t}, t \in \mathbf{T}\right\}$ be a family of joint Gaussian random variables for which $E \xi_{t}=0$ (e.g., $\xi_{t}, t \in \mathbf{T}$, is a Gaussian stochastic process).

The space $S G_{\Xi}(\Omega)$ is the space of square Gaussian random variables if any element $\eta \in S G_{\Xi}(\Omega)$ can be presented as

$$
\eta=\bar{\xi}^{\top} A \bar{\xi}-\mathbf{E} \bar{\xi}^{\top} A \bar{\xi}
$$

where $\bar{\xi}^{\top}=\left(\xi_{1}, \xi_{2}, \ldots, \xi_{n}\right), \xi_{k} \in \Xi, k=1, \ldots, n, A$ is a real-valued matrix or the element $\eta \in S G_{\Xi}(\Omega)$ is the square mean limit of the sequence (50)

$$
\eta=\operatorname{lii.m}_{\cdot n \rightarrow \infty}\left(\bar{\xi}_{n}^{\top} A \bar{\xi}_{n}-\mathbf{E} \bar{\xi}_{n}^{\top} A \bar{\xi}_{n}\right) .
$$

Definition 4.2. [7] A stochastic process $\xi(t)=\{\xi(t), t \in \mathbf{T}\}$ is square Gaussian if for any $t \in \mathbf{T}$ a random variable $\xi(t)$ belongs to the space $S G_{\Xi}(\Omega)$.

There are shown in the book by Buldygin and Kozachenko [7] that

- $S G_{\Xi}(\Omega)$ is a Banach space with respect to the norm $\|\zeta\|=\sqrt{\mathbf{E} \zeta^{2}}$;

- $S G_{\Xi}(\Omega)$ is a subspace of the Orlicz space $L_{U}(\Omega)$ generated by the function

$$
U(x)=\exp |x|-1 ;
$$

- the norm $\|\zeta\|_{L_{U}(\Omega)}$ on $S G_{\Xi}(\Omega)$ is equivalent to the norm $\|\zeta\|$.

Example 4.1. Consider a family of Gaussian centered stochastic processes $\xi_{1}(t), \xi_{2}(t), \ldots, \xi_{n}(t), t \in \mathbf{T}$. Let the matrix $A(t)$ be symmetric. Then

$$
X(t)=\bar{\xi}^{\top}(t) A(t) \bar{\xi}(t)-\mathbf{E} \bar{\xi}^{\top}(t) A(t) \bar{\xi}(t),
$$

where $\bar{\xi}^{\top}(t)=\left(\xi_{1}(t), \xi_{2}(t), \ldots, \xi_{n}(t)\right)$, is a square Gaussian stochastic process.

For more information about properties of square Gaussian random processes see [16], [17], [18], [7], [20]. 


\subsection{An estimate for the $C(T)$ norm of a square Gaussian stochastic process}

Denote by $N(u)$ the metric massiveness, that is the least number of closed balls of radius $u$, covering the set $\mathbf{T}$ with respect to the metric $\rho$. Let $\xi(t)=\{\xi(t), t \in \mathbf{T}\}$ be a square Gaussian stochastic process. Assume that there exists a monotonically increasing continuous function $\sigma(h), h>0$, such that $\sigma(h) \rightarrow 0$ as $h \rightarrow 0$, and the inequality

$$
\sup _{\rho(t, s) \leq h}(\operatorname{Var}(\xi(t)-\xi(s)))^{\frac{1}{2}} \leq \sigma(h)
$$

holds true.

Define now the following values:

$$
\begin{gathered}
\varepsilon_{0}=\inf _{t \in \mathbf{T}} \sup _{s \in \mathbf{T}} \rho(t, s), \quad t_{0}=\sigma\left(\varepsilon_{0}\right), \\
\gamma_{0}=\sup _{t \in \mathbf{T}}(\operatorname{Var} \xi(t))^{1 / 2},
\end{gathered}
$$

Let $C$ be a maximum of $t_{0}$ and $\gamma_{0}, C=\max \left\{t_{0}, \gamma_{0}\right\}$. The next theorem gives an estimate for the large deviation probability of square Gaussian process in the norm of continuous function. The proof of the Theorem can be found in the article [19].

Theorem 4.1

Let $\xi(t)=\{\xi(t), t \in \mathbf{T}\}$ be a separable square Gaussian stochastic process. Suppose that there exists an increasing function $r(u) \geq 0, u \geq 1$, with the properties: $r(u) \rightarrow \infty$ and $u \rightarrow \infty$ and let the function $r(\exp \{t\})$ be convex. Assume that the following integral

$$
\int_{0}^{t_{0}} r\left(N\left(\sigma^{(-1)}(u)\right)\right) d u
$$

is convergent. Then for all $x>0$

$$
\begin{aligned}
\mathbf{P}\left\{\sup _{t \in \mathbf{T}}|\xi(t)|>x\right\} \leq 2 \inf _{0<p<1} & \left\{r^{(-1)}\left(\frac{1}{t_{0} p} \int_{0}^{t_{0} p} r\left(N\left(\sigma^{(-1)}(\nu)\right)\right) d \nu\right)\right. \\
\times & \left.\left(1+\frac{\sqrt{2} x(1-p)}{C}\right)^{\frac{1}{2}} \exp \left\{-\frac{x(1-p)}{\sqrt{2} \gamma_{0}}\right\}\right\} .
\end{aligned}
$$

\subsection{An estimate for the $L_{p}(T)$ norm of a square Gaussian stochastic process}

The following theorem can be found in article [21].

\section{Theorem 4.2}

[21] Let $\{T, \mathfrak{A}, \mu\}$ be a measurable space, where $\mathbf{T}$ is a parametric set, and let $\xi=\{\xi(t), t \in \mathbf{T}\}$ be a measurable square Gaussian stochastic process. Assume that the Lebesgue integral $\int_{\mathbf{T}}\left(\mathbf{E} \xi^{2}(t)\right)^{\frac{p}{2}} d \mu(t)$ is well defined for $p \geq 1$. Then the integral $\int_{\mathbf{T}}\left(\mathbf{E} \xi^{2}(t)\right)^{p} d \mu(t)$ exists with probability 1 , and

$$
P\left\{\int_{\mathbf{T}}|\xi(t)|^{p} d \mu(t)>x\right\} \leq 2 \sqrt{1+\frac{x^{1 / p} \sqrt{2}}{C_{p}^{\frac{1}{p}}}} \exp \left\{-\frac{x^{1 / p}}{\sqrt{2} C_{p}^{\frac{1}{p}}}\right\},
$$

for all $x \geq\left(\frac{p}{\sqrt{2}}+\sqrt{\left(\frac{p}{2}+1\right) p}\right)^{p} C_{p}$, where $C_{p}=\int_{\mathbf{T}}\left(\mathbf{E} \xi^{2}(t)\right)^{\frac{p}{2}} d \mu(t)$.

\section{On the rate of convergence of the estimator of impulse response function}

This section is devoted to the investigation of the rate of convergence of estimators of unknown impulse response function in the space of continuous functions and in the space $L_{2}([0, A])$. 
Suppose that $X_{N, i}=\left(X_{N, i}(u), u \in \mathbb{R}\right), i=\overline{1, n}$, are real-valued independent centered Gaussian processes from (23). Assume that they perturb a time-invariant casual continuous linear system (1).

Consider estimator (6) for the impulse response function $L$. The output process $Y(t)$ is defined by (24).

The next Lemma is clear.

Lemma 5.1

Stochastic process $\hat{Z}_{N, n}(\tau)=\hat{L}_{N, n}(\tau)-E \hat{L}_{N, n}(\tau), \tau>0$, is a square Gaussian one.

Consider a difference of the estimator $\hat{L}_{N, n}(\tau)$ and the impulse function $L(\tau)$

$$
L(\tau)-\hat{L}_{N, n}(\tau), \quad \tau>0 .
$$

At first we study the distribution of supremum for this difference on the domain $[0, A]$, where $A$ is a fixed positive number,

$$
P\left\{\sup _{\tau \in[0, A]}\left|\hat{L}_{N, n}(\tau)-L(\tau)\right| \geq \varepsilon\right\}, \quad \varepsilon>0 .
$$

Denote

$$
l_{N, n}^{*}=\frac{2 K^{2}}{3 \sqrt{N+1}} I_{L} .
$$

Then from (26) it follows that

$$
\left|E \hat{L}_{N, n}(\tau)-L(\tau)\right| \leq l_{N, n}^{*}, \quad \tau \in[0, A] .
$$

Put

$$
\gamma_{0}(N, n)=\gamma_{0}=\frac{K^{4} I_{L}^{2}}{n}\left(N \frac{N^{2}-1}{2 N^{2}}+\frac{(2 \sqrt{N}-2)^{2}}{N}\right) .
$$

From (34) we have that

$$
\sup _{\tau \in[0, A]} \operatorname{Var} \hat{Z}_{N, n}(\tau) \leq \gamma_{0}
$$

Let

$$
M_{\alpha}=2^{2-\frac{1}{2 \alpha}} e^{1 / \alpha} \gamma_{0}^{-\frac{1}{2}-\frac{1}{\alpha}} \alpha^{1 / \alpha-1 / 2} .
$$

The following theorem gives the rate of convergence of the impulse function estimator in the space of continuous function.

Theorem 5.1

Suppose that the conditions A, B are fulfilled. Then the inequality

$$
\begin{aligned}
& P \quad\left\{\sup _{\tau \in[0, A]}\left|L(\tau)-\hat{L}_{N, n}(\tau)\right|>\varepsilon\right\} \leq M_{\alpha}\left(\varepsilon-l_{N, n}^{*}\right)^{\frac{1}{\alpha}} \\
& \times \quad\left(C \alpha+\sqrt{2} \alpha\left(\varepsilon-l_{N, n}^{*}\right)-2 \gamma_{0}\right)^{\frac{1}{2}} \exp \left\{-\frac{\varepsilon-l_{N, n}^{*}}{\sqrt{2} \gamma_{0}}+\frac{1}{\alpha}\right\}
\end{aligned}
$$

holds true for

$$
\varepsilon>\frac{\sqrt{2} \gamma_{0}}{\alpha}+l_{N, n}^{*}, \quad \alpha \in(0,1]
$$

Proof

The difference $L(\tau)-\hat{L}_{N, n}(\tau)$ can be presented as

$$
L(\tau)-\hat{L}_{N, n}(\tau)=\left(L(\tau)-E \hat{L}_{N, n}(\tau)\right)-\left(\hat{L}_{N, n}(\tau)-E \hat{L}_{N, n}(\tau)\right)=l_{N, n}(\tau)-\hat{Z}_{N, n}(\tau) .
$$

Then

$$
L(\tau)-\hat{L}_{N, n}(\tau) \geq \varepsilon \quad \Leftrightarrow \quad \hat{Z}_{T}(\tau) \leq-\left(\varepsilon-l_{N, n}(\tau)\right),
$$




$$
L(\tau)-\hat{L}_{N, n}(\tau) \leq-\varepsilon \quad \Leftrightarrow \quad \hat{Z}_{T}(\tau) \geq+\varepsilon+l_{N, n}(\tau) .
$$

Hence, for $\varepsilon>l_{N, n}^{*}$ we obtain

$$
\left\{\left|L(\tau)-\hat{L}_{N, n}(\tau)\right| \geq \varepsilon\right\} \subset\left\{\left|\hat{Z}_{T}(\tau)\right| \geq \varepsilon-l_{N, n}^{*}\right\}
$$

and

$$
P\left\{\sup _{\tau \in[0, A]}\left|L(\tau)-\hat{L}_{N, n}(\tau)\right| \geq \varepsilon\right\} \leq P\left\{\sup _{\tau \in[0, A]}\left|\hat{Z}_{T}(\tau)\right| \geq \varepsilon-l_{N, n}^{*}\right\} .
$$

In the frame of proving put

$$
x=\varepsilon-l_{N, n}^{*} .
$$

Since from Lemma 5.1 follows that $\hat{Z}_{T}(\tau)$ is a square Gaussian process then Theorem 4.1 can be used. It follows from (48) that as a function $\sigma(h)$ the function $\sigma(h)=C_{g}(N, n) \cdot h^{\alpha}$ could be considered, where $C_{g}(N, n)$ is defined in (49). In this case the inverse function equals $\sigma^{(-1)}(h)=\left(\frac{h}{C_{g}(N, n)}\right)^{1 / \alpha}$. The metric massiveness of the segment $[0, A]$ with respect to the metric $\rho(t, s)=|t-s|$ can be majorized as

$$
N(u) \leq \frac{A}{2 u}+1
$$

Therefore,

$$
\begin{aligned}
N\left(\sigma^{(-1)}(u)\right) & \leq\left(\frac{A}{2 \sigma^{(-1)}(u)}+1\right) \\
& =\left(\frac{A}{2}\left(\frac{C_{g}(N, n)}{u}\right)^{1 / \alpha}+1\right) .
\end{aligned}
$$

Consider the function $r(u)=u^{\beta}-1$, where $\beta \in(0, \alpha)$. It's clear that the conditions of Theorem 4.1 for the function $r(u)$ are satisfied. Since $0<p<1$ and $t_{0}=C_{g}(N, n)\left(\frac{A}{2}\right)^{\alpha}$ then $\frac{A}{2}\left(\frac{C_{g}(N, n)}{p t_{0}}\right)^{1 / \alpha}>1$. Hence, it follows from the assumption $0<u<t_{0} p$ that the inequality

$$
N\left(\sigma^{(-1)}(u)\right) \leq A\left(\frac{C_{g}(N, n)}{u}\right)^{1 / \alpha}
$$

holds. Since the inverse function of $r(u)$ is $r^{(-1)}(u)=(u+1)^{1 / \beta}$, then

$$
\begin{aligned}
r^{(-1)}\left(\frac{1}{t_{0} p} \int_{0}^{t_{0} p} r\left(N\left(\sigma^{(-1)}(\nu)\right)\right) d \nu\right) & =\left(\frac{1}{t_{0} p} \int_{0}^{t_{0} p}\left[\left(\frac{A}{2}\left(\frac{C_{g}(N, n)}{u}\right)^{1 / \alpha}+1\right)^{\beta}\right] d u\right)^{1 / \beta} \\
& \leq\left(\frac{1}{t_{0} p} \int_{0}^{t_{0} p}\left[A\left(\frac{C_{g}(N, n)}{u}\right)^{1 / \alpha}\right]^{\beta} d u\right)^{1 / \beta} \\
& =2\left(\frac{\alpha}{\alpha-\beta}\right)^{1 / \beta} p^{-1 / \alpha} .
\end{aligned}
$$

Find the minimum with respect to $\beta$ of the right-hand side of the relationship above

$$
\inf _{\beta \in(0, \alpha)}\left(\frac{\alpha}{\alpha-\beta}\right)^{1 / \beta}=\lim _{\beta \rightarrow 0}\left(\frac{1}{1-\beta / \alpha}\right)^{1 / \beta}=e^{1 / \alpha} .
$$

From inequality (51) and equity above follows that for $x>0$

$$
\begin{aligned}
& P\left\{\sup _{\tau \in[0, A]}\left|\hat{Z}_{T}(\tau)\right|>x\right\} \leq \\
& \leq 4 e^{1 / \alpha} C^{-\frac{1}{2}} \inf _{0<p<1}\left\{\frac{\sqrt{C+\sqrt{2} x(1-p)}}{p^{1 / \alpha}} \exp \left\{-\frac{x(1-p)}{\sqrt{2} \gamma_{0}}\right\}\right\} .
\end{aligned}
$$


The minimum point of right-hand side of (56) is

$$
p_{\min }=\frac{\sqrt{2} \gamma_{0}}{\alpha x} .
$$

Since by the condition of Theorem $p \in(0,1)$, then for

$$
x>\frac{\sqrt{2} \gamma_{0}}{\alpha}
$$

the value $p_{\min }$ can be substituted in (56) and the following inequality is obtained

$$
\begin{aligned}
& P\left\{\sup _{\tau \in[0, A]}\left|\hat{Z}_{T}(\tau)\right|>x\right\} \leq \\
& \leq M_{\alpha} x^{\frac{1}{\alpha}} \sqrt{C \alpha+\sqrt{2} \alpha x-2 \gamma_{0}} \exp \left\{-\frac{x}{\sqrt{2} \gamma_{0}}+\frac{1}{\alpha}\right\},
\end{aligned}
$$

where the constant $M_{\alpha}$ equals

$$
M_{\alpha}=2^{2-\frac{1}{2 \alpha}} e^{1 / \alpha} \gamma_{0}^{-\frac{1}{2}-\frac{1}{\alpha}} \alpha^{1 / \alpha-1 / 2} .
$$

Taking into account that $x=\varepsilon-l_{N, n}^{*}$, the assertion of Theorem follows from (57).

Theorem 4.2 can be used to obtain the following estimate for the cross-correlogram in the space $L_{p}([0, A])$, where the constant $A>0$ is fixed.

Consider a parametric set $\mathbf{T}=[0, A]$ and let $\{[0, A], \mathfrak{A}, \mu\}$ be a metric space with Euclidean measure $\mu$.

Theorem 5.2

Assume that the conditions $\mathbf{A}$ and $\mathbf{B}$ are satisfied. Then for

$$
\varepsilon \geq\left(\left(\frac{p}{\sqrt{2}}+\sqrt{\left(\frac{p}{2}+1\right) p}\right) A^{\frac{1}{p}} \gamma_{0}^{\frac{1}{2}}+A l_{N, n}^{*}\right)^{p}
$$

the estimate

$$
P\left\{\int_{0}^{A}\left|L(\tau)-\hat{L}_{N, n}(\tau)\right|^{p} d \tau>\varepsilon\right\} \leq 2 \sqrt{1+\frac{\left(\varepsilon^{\frac{1}{p}}-A l_{N, n}^{*}\right) \sqrt{2}}{A^{\frac{1}{p}} \gamma_{0}^{\frac{1}{2}}}} \exp \left\{-\frac{\varepsilon^{\frac{1}{p}}-A l_{N, n}^{*}}{\sqrt{2} A^{\frac{1}{p}} \gamma_{0}^{\frac{1}{2}}}\right\}
$$

holds true.

Proof

We show first that the result of Theorem 4.2 can be applied to the process $\hat{Z}_{N, n}(\tau), \tau>0$. Really, by Lemma 5.1 $\hat{Z}_{N, n}(\tau)$ is a square Gaussian stochastic process. Prove that the Lebesgue integral

$$
\int_{0}^{A}\left(\mathbf{E} \hat{Z}_{N, n}^{2}(\tau)\right)^{\frac{p}{2}} d \mu(\tau)
$$

is correctly defined. From inequality (27) follows that

$$
\begin{aligned}
\int_{0}^{A}\left(\mathbf{E} \hat{Z}_{N, n}^{2}(\tau)\right)^{\frac{p}{2}} d \mu(\tau) & =\int_{0}^{A}\left(\operatorname{Var} \hat{Z}_{N, n}(\tau)\right)^{\frac{p}{2}} d \tau \\
& <A\left(\frac{K^{4} I_{L}^{2}}{n}\left(N \frac{N^{2}-1}{2 N^{2}}+\frac{(2 \sqrt{N}-2)^{2}}{N}\right)\right)^{\frac{p}{2}}
\end{aligned}
$$


Therefore, inequality (52) for the process $\hat{Z}_{N, n}(\tau)$ as $x \geq\left(\frac{p}{\sqrt{2}}+\sqrt{\left(\frac{p}{2}+1\right) p}\right)^{p} C_{p}$ can be rewritten as

$$
P\left\{\int_{0}^{A}\left|\hat{Z}_{N, n}(\tau)\right|^{p} d \mu(\tau)>x\right\} \leq 2 \sqrt{1+\frac{x^{1 / p} \sqrt{2}}{C_{p}^{\frac{1}{p}}}} \exp \left\{-\frac{x^{1 / p}}{\sqrt{2} C_{p}^{\frac{1}{p}}}\right\},
$$

where $C_{p}=\int_{0}^{A}\left(\mathbf{E} \hat{Z}_{N, n}^{2}(\tau)\right)^{\frac{p}{2}} d \tau$.

From the Minkowski inequality follows that

$$
\begin{aligned}
\left(\int_{0}^{A}\left|L(\tau)-\hat{L}_{N, n}(\tau)\right|^{p} d \mu(\tau)\right)^{\frac{1}{p}} & =\left(\int_{0}^{A}\left|L(\tau) \pm \mathbf{E} \hat{L}_{N, n}(\tau)-\hat{L}_{N, n}(\tau)\right|^{p} d \mu(\tau)\right)^{\frac{1}{p}} \\
& \leq\left(\int_{0}^{A}\left|L(\tau)-\mathbf{E} \hat{L}_{N, n}(\tau)\right|^{p} d \mu(\tau)\right)^{\frac{1}{p}} \\
& +\left(\int_{0}^{A}\left|\mathbf{E} \hat{L}_{N, n}(\tau)-\hat{L}_{N, n}(\tau)\right|^{p} d \mu(\tau)\right)^{\frac{1}{p}} \\
& \leq l_{N, n}^{*} \cdot A+\left(\int_{0}^{A}\left|\hat{Z}_{N, n}(\tau)\right|^{p} d \mu(\tau)\right)^{\frac{1}{p}}
\end{aligned}
$$

Since from the relationship above follows that

$$
\begin{aligned}
\left\{\int_{0}^{A}\left|L(\tau)-\hat{L}_{N, n}(\tau)\right|^{p} d \mu(\tau)>\varepsilon\right\} & =\left\{\left(\int_{0}^{A}\left|L(\tau)-\hat{L}_{N, n}(\tau)\right|^{p} d \mu(\tau)\right)^{\frac{1}{p}}>\varepsilon^{\frac{1}{p}}\right\} \\
& \subset\left\{A l_{N, n}^{*}+\left(\int_{0}^{A}\left|\hat{Z}_{N, n}(\tau)\right|^{p} d \mu(\tau)\right)^{\frac{1}{p}}>\varepsilon^{\frac{1}{p}}\right\} \\
& =\left\{\int_{0}^{A}\left|\hat{Z}_{N, n}(\tau)\right|^{p} d \mu(\tau)>\left(\varepsilon^{\frac{1}{p}}-A l_{N, n}^{*}\right)^{p}\right\},
\end{aligned}
$$

then substituting $x=\left(\varepsilon^{\frac{1}{p}}-A l_{N, n}^{*}\right)^{p}$ in (59), we obtain

$$
P\left\{\int_{0}^{A}\left|L(\tau)-\hat{L}_{N, n}(\tau)\right|^{p} d \mu(\tau)>\varepsilon\right\} \leq 2 \sqrt{1+\frac{\left(\varepsilon^{\frac{1}{p}}-A l_{N, n}^{*}\right) \sqrt{2}}{C_{p}^{\frac{1}{p}}}} \exp \left\{-\frac{\varepsilon^{\frac{1}{p}}-A l_{N, n}^{*}}{\sqrt{2} C_{p}^{\frac{1}{p}}}\right\} .
$$

Since (34) implies $\sup _{\tau \in[0, A]} \operatorname{Var} \hat{Z}_{N, n}(\tau) \leq \gamma_{0}$, where $\gamma_{0}$ is from (53), then

$$
C_{p}=\int_{0}^{A}\left(\mathbf{E} \hat{Z}_{N, n}^{2}(\tau)\right)^{\frac{p}{2}} d \tau \leq A \gamma_{0}^{\frac{p}{2}}
$$

To complete the proof it's enough to substitute the above value $C_{p}$ in (60). Hence,

$$
P\left\{\int_{0}^{A}\left|L(\tau)-\hat{L}_{N, n}(\tau)\right|^{p} d \mu(\tau)>\varepsilon\right\} \leq 2 \sqrt{1+\frac{\left(\varepsilon^{\frac{1}{p}}-A l_{N, n}^{*}\right) \sqrt{2}}{A^{\frac{1}{p}} \gamma_{0}^{\frac{1}{2}}}} \exp \left\{-\frac{\varepsilon^{\frac{1}{p}}-A l_{N, n}^{*}}{\sqrt{2} A^{\frac{1}{p}} \gamma_{0}^{\frac{1}{2}}}\right\}
$$




\section{Testing hypotheses on the impulse response function}

Using Theorem 5.1 and Theorem 5.2 it is possible to test hypothesis on the shape of impulse response function.

Let the null hypothesis $\mathbf{H}_{\mathbf{0}}$ state that an impulse response function is $L(\tau), \tau \in[0, A]$, and the alternative $\mathbf{H}_{\mathbf{a}}$ implies the opposite statement.

Denote

$$
g_{1}(\varepsilon)=M_{\alpha}\left(\varepsilon-l_{N, n}^{*}\right)^{\frac{1}{\alpha}}\left(C \alpha+\sqrt{2} \alpha\left(\varepsilon-l_{N, n}^{*}\right)-2 \gamma_{0}\right)^{\frac{1}{2}} \exp \left\{-\frac{\varepsilon-l_{N, n}^{*}}{\sqrt{2} \gamma_{0}}+\frac{1}{\alpha}\right\} .
$$

From Theorem 5.1 follows that if $\varepsilon>z_{N, n}=\frac{\sqrt{2} \gamma_{0}}{\alpha}+l_{N, n}^{*}, \quad \alpha \in(0,1]$, then

$$
P\left\{\sup _{\tau \in[0, A]}\left|L(\tau)-\hat{L}_{N, n}(\tau)\right|>\varepsilon\right\} \leq g_{1}(\varepsilon) .
$$

Let $\varepsilon_{1, \delta}$ be a solution of the equation

$$
g_{1}\left(\varepsilon_{1, \delta}\right)=\delta, \quad 0<\delta<1
$$

Put

$$
\varepsilon_{1, \delta}^{*}=\max \left\{\varepsilon_{1, \delta}, z_{N, n}\right\}
$$

It is obvious that $g_{1}\left(\varepsilon_{1, \delta}^{*}\right) \leq \delta$ and

$$
P\left\{\sup _{\tau \in[0, A]}\left|L(\tau)-\hat{L}_{N, n}(\tau)\right|>\varepsilon_{1, \delta}^{*}\right\} \leq \delta .
$$

From Theorem 5.1 it follows that to test the hypothesis $\mathbf{H}_{\mathbf{0}}$, we can use the following criterion.

Criterion 1. For a given level of confidence $1-\delta, \delta \in(0,1)$, the hypothesis $\mathbf{H}_{0}$ is rejected if

$$
\sup _{\tau \in[0, A]}\left|L(\tau)-\hat{L}_{N, n}(\tau)\right|>\varepsilon_{1, \delta}^{*},
$$

otherwise the hypothesis $\mathbf{H}_{\mathbf{0}}$ is accepted, where $\varepsilon_{1, \delta}^{*}$ is from (61).

Remark 6.1. The equation $g_{1}\left(\varepsilon_{1, \delta}\right)=\delta$, has a solution for any $\delta>0$ since the function $g_{1}(\varepsilon)$ decreases. We can find solution of the equation using numerical methods.

Denote

$$
g_{2}(\varepsilon)=2 \sqrt{1+\frac{\left(\varepsilon^{\frac{1}{p}}-A l_{N, n}^{*}\right) \sqrt{2}}{A^{\frac{1}{p}} \gamma_{0}^{\frac{1}{2}}}} \exp \left\{-\frac{\varepsilon^{\frac{1}{p}}-A l_{N, n}^{*}}{\sqrt{2} A^{\frac{1}{p}} \gamma_{0}^{\frac{1}{2}}}\right\} .
$$

From Theorem 5.2 follows that if

$$
\varepsilon>t_{N, n}(p)=\left(\left(\frac{p}{\sqrt{2}}+\sqrt{\left.\left(\frac{p}{2}+1\right) p\right)} A^{\frac{1}{p}} \gamma_{0}^{\frac{1}{2}}+A l_{N, n}^{*}\right)^{p},\right.
$$

then

$$
P\left\{\int_{0}^{A}\left|L(\tau)-\hat{L}_{N, n}(\tau)\right|^{p} d \tau>\varepsilon\right\} \leq g_{2}(\varepsilon) .
$$

Let $\varepsilon_{2, \delta}$ be a solution of the equation $g_{2}\left(\varepsilon_{2, \delta}\right)=\delta, 0<\delta<1$. Put

$$
\varepsilon_{2, \delta}^{*}=\max \left\{\varepsilon_{2, \delta}, t_{N, n}(p)\right\} .
$$


It is clear that $g_{2}\left(\varepsilon_{2, \delta}^{*}\right) \leq \delta$ and

$$
P\left\{\sup _{\tau \in[0, A]}\left|L(\tau)-\hat{L}_{N, n}(\tau)\right|>\varepsilon_{2, \delta}^{*}\right\} \leq \delta .
$$

From Theorem 5.2 it follows that to test the hypothesis $\mathbf{H}_{\mathbf{0}}$, we can use the following criterion.

Criterion 2. For a given level of confidence $1-\delta, \delta \in(0,1)$, the hypothesis $\mathbf{H}_{\mathbf{0}}$ is rejected if

$$
\int_{0}^{A}\left|L(\tau)-\hat{L}_{N, n}(\tau)\right|^{p} d \tau>\varepsilon_{2, \delta}^{*}
$$

otherwise the hypothesis $\mathbf{H}_{\mathbf{0}}$ is accepted, where $\varepsilon_{2, \delta}^{*}$ is from (62).

\section{Conclusions}

In this paper we considere time-invariant continuous linear system in which the impulse response function was estimated applying a new proposed method. The input signal process is supposed to be a zero mean Gaussian stochastic process which is represented as a series with respect to an orthonormal basis in $L_{2}(\mathbf{R})$. A particular case where the orthonormal basis is given by the Hermite functions is studied in details. Some characteristics of the estimator of impulse function such as mathematical expectation, variance and variance of the increments are described. We also investigated the convergence rate for the estimator of unknown impulse response function in the space of continuous functions and in the space $L_{p}([0, A])$. For this reason the theory of square Gaussian random variables and processes is applied, namely we use inequalities for the $C(T)$ and $L_{p}(T)$ norms of square Gaussian stochastic process. It gives us an opportunity to construct two criteria of testing hypothesis on the shape of the impulse response function.

\section{REFERENCES}

1. H. Akaike, On the statistical estimation of the frequence response function of a system having multiple input, Ann. Inst. Statist. Math., vol. 17, pp. 185-210, 1965.

2. H. Akaike, On the use of non-Gaussian process in the identification of a linear dynamic system, Ann. Inst. Statist. Math., vol. 18, pp. 269-276, 1966.

3. J. S. Bendat, and A.G. Piersol, Engineering Applications of Correlation and Spectral Analysis, Wiley, New York, 1980.

4. I.P. Blazhievska, Asymptotic unbiasedness and consystency of cross-correlogram estimator for impulse response functions of linear system, Naukovi Visti NTUU KPI? vol. 4, pp. 7-12, 2014.(in Ukrainian)

5. V.V. Buldygin, and I.P. Blazhievska, On correlation properties of the cross-correlogram estimators of impulse response functions, Naukovi Visti NTUU KPI? vol. 5, pp. 120-128, 2009.(in Ukrainian)

6. V.V. Buldygin, and I.P. Blazhievska, Asymptotic properties of cross-correlogram estimator for impulse response functions of linear system, Naukovi Visti NTUU KPI? vol. 4, pp. 16-27, 2010.(in Ukrainian)

7. V.V. Buldygin, and Yu.V. Kozachenko, Metric characterization of random variables and random processes, Amer. Math. Soc., Providence, RI, 2000.

8. V.V. Buldygin, and V.G. Kurotschka, On cross-coorrelogram estimators of the response function in continuous linear systems from discrete observations, Random Oper. and Stoch. Equ., vol.7, no. 1, pp. 71-90, 1999.

9. V.V. Buldygin, and $\mathrm{Fu} \mathrm{Li}$, On asymptotic normality of an estimation of unit impulse responses of linear system I Theor. Probability and Math. Statist., vol. 54, pp. 3-17, 1997.

10. V.V. Buldygin, and $\mathrm{Fu} \mathrm{Li,} \mathrm{On} \mathrm{asymptotic} \mathrm{normality} \mathrm{of} \mathrm{an} \mathrm{estimation} \mathrm{of} \mathrm{unit} \mathrm{impulse} \mathrm{responses} \mathrm{of} \mathrm{linear} \mathrm{system} \mathrm{II} \mathrm{Theor.} \mathrm{Probability}$ and Math. Statist., vol. 55, pp. 30-37, 1997.

11. V. Buldygin, L. Utzet, and V. Zaiats, Asymptotic normality of cross-coorrelogram estimators of the response function, Statistical Infernce for Stochastic Processes, vol. 7, pp. 1-34, 2004.

12. V. Buldygin, L. Utzet, and V. Zaiats, A note on the application of intergals involving cyclic products of kernels, QESTIIO, vol. 26, no. $1-2$, pp. $3-14,2002$.

13. A. Erdelyi, Higher transcendental functions, Mc Graw-Hill Book Company, Inc, 1953.

14. I.I. Gikhman, and A.V. Skorokhod, Introduction to the theory of random processes, Dover Publications Inc., Mineola, New York,1996.

15. E. J. Hannan, and M. Deistler, The Statistical Theory of Linear Systems, Wiley, New York, 1988.

16. Yu. V. Kozachenko, and O.M. Moklyachuk, Large deviation probabilities for square-Gaussian stochastic processes, Extremes, vol. 2 , no. 3, pp. 269-293, 1999. 
17. Yu.V Kozachenko, and O.M. Moklyachuk, Square-Gaussian stochastic processes, Theory of Stoch. Processes, vol. 6(22), no.3-4, pp. 98-121, 2000.

18. Yu.V. Kozachenko, A.O. Pashko, and I.V. Rozora, Simulation of stochastic processes and fields, Zadruga, Kyiv, 2007. (in Ukrainian)

19. Yu. Kozacenko, and I. Rozora, On cross-correlogram estimators of impulse response function, Theor. Probability and Math. Statist., vol. 93, pp. 75-83, 2015.

20. Yu.V. Kozachenko, and O.V. Stus, Square-Gaussian random processes and estimators of covariance functions, Math. Communications., vol. 3, no. 1, pp. 83-94, 1998.

21. Yu. Kozachenko, and V. Troshki, A criterion for testing hypotheses about the covariance function of a stationary Gaussian stochastic process, Modern Stochastics: Theory and Applications, vol. 1, no. 1, pp. 139-149, 2014.

22. M. Schetzen, The Volterra and Wiener Theories of Nonlinear Systems, Wiley, New York, 1980.

23. T. Söderström, and P. Stoica, System Identification, Prentice-Hall, London, 1989. 